\title{
Fractalidad en regiones de Formación Estelar
}

\author{
H.E. Caicedo-Ortiz*1, H.O. Castañeda ${ }^{2}$, E. Santiago-Cortés ${ }^{1,3}$ \\ ${ }^{1}$ Corporación Universitaria Autónoma del Cauca, Popayán, Colombia \\ ${ }^{2}$ Escuela Superior de Física y Matemáticas, Instituto Politécnico Nacional, México \\ ${ }^{3}$ Escuela Nacional de Ciencias Biológicas y Centro de Nanociencias y Micro y Nanotecnologías, Instituto Politécnico \\ Nacional, México
}

Received on January 7, 2017. Revised on February 8, 2017. Accepted on February 12, 2017.

\begin{abstract}
En este artículo se calcula la dimensión de Hausdorff-Besicovitch ó dimensión fractal de dos objetos astronómicos, utilizando el método de conteo de cajas. Se utilizaron imágenes obtenidas con el Telescopio Espacial Hubble de las regiones de formación estelar conocidas como Hubble V y Hubble X, localizadas en la galaxia NGC 6822. Los valores de dimensión fractal de estas regiones HII gigantes son comparados con los resultados de otros objetos astronómicos de la misma especie reportados en la literatura. Sugerimos un nuevo esquema de clasificación de regiones HII gigantes basado en esta propiedad.
\end{abstract}

Palabras clave: Dimensión fractal; regiones HII gigantes, Hubble V, Hubble X, NGC 6822.

This paper calculates the Hausdorff-Besicovitch dimension or fractal dimension of two astronomical objects using the box counting method. Images were obtained with the Hubble Space Telescope from the regions of star formation known as Hubble V and Hubble X, located in the NGC 6822 galaxy. The fractal dimension values for these H II giant regions are compared to the results of other astronomical objects from the same species that are reported in the literature. A new scheme for classification of the giant H II regions is proposed based on this property.

Keywords: Fractal dimension; giant HII regions, Hubble V, Hubble X, NGC 6822.

\section{Introducción}

Un sistema complejo se puede definir como una estructura constituida por un determinado número de elementos, que interaccionan entre sí bajo ciertas reglas, generando propiedades emergentes, información adicional no visible antes por el observador, las cuales no pueden explicarse a partir de las características de los elementos aislados [1].

La dimensión de un objeto representa la medida de su grado de complejidad. Cuando esta medida no es un número entero, se interpreta que el objeto es un fractal 1, 2.

Mandelbrot introduce el concepto de fractalidad [2], encontrando un uso práctico a estas estructuras matemáticas. En la actualidad, la geometría fractal y multifractal abarca áreas tan diversos como la

*Endereço de correspondência: hernan-
do.caicedo@uniautonoma.edu.co. física, la biología, la medicina, la informática, y la economía.

En el campo de la astrofísica [3], con la excepción de la estructura a gran escala del universo (una área en la cual el concepto de una organización jerárquica fue introducido mucho antes de crearse la geometría fractal de Mandelbrot [4 6]), su uso como una herramienta de estudio en el campo ha sido limitado $7-17$.

Un análisis fractal es apropiado para estudiar sistemas jerárquicos y autosimilares, siendo la dimensión fractal el parámetro que cuantifica, para las regiones de formación estelar, la heterogenidad espacial en la distribución del gas y las estrellas. Este parámetro permite realizar estudios entre objetos similares empleando el lenguaje de la morfología matemática [18], ofreciendo una cuantificación precisa de formulaciones lingüísticas que de modo vago son empleadas en astronomía [19. 
El objetivo de este trabajo es determinar la dimensión fractal de dos regiones HII gigantes como son Hubble V y Hubble X. Empleando resultados de estudios previos de dimensión fractal de otras regiones HII, se presenta un nuevo esquema de clasificación de las regiones HII gigantes, basado en su dimensión fractal.

\section{Dimensión Fractal}

Una medida del grado de complejidad de un sistema es expresándolo como una "dimensión". La idea de dimensión caracteriza los espacios euclidianos en los que se establece el concepto de medida [20]. La dimensión espacial habitualmente conocida presenta un valor entero. La dimensión fractal, a diferencia de la dimensión espacial, no es un número entero.

Una propiedad importante de la geometría fractal es que permite una caracterización de la irregularidad a diferentes escalas que la geometría euclidiana clásica no permite.

En 1919, Hausdorff [21] desarrolló la teoría matemática que permite describir objetos complejos dentro de un espacio de dimensión no nula (métrica de Hausdorff). Besicovitch en 1920 utilizó la dimensión de Hausdorff para medir y dimensionar estos conjuntos 22 . En 1957, Hurst 23 enunció la ley que estudia el efecto de la invarianza al cambio de escala en series temporales y que presentan memoria a largo plazo. La invarianza al cambio de escala es la propiedad cualitativa fundamental para definir un conjunto fractal y su expresión matemática es la ley de potencias. Ambos comportamientos aparecen tanto en series espaciales (geometría y forma) como en series temporales (distribución de un fenómeno a lo largo del tiempo).

El análisis fractal ofrece una herramienta que permite caracterizar de forma geométrica estructuras y propiedades de entidades complejas, a partir del cálculo de la dimensión fractal. La generalización de la dimensión geométrica nos permite discernir entre estructuras en función de la complejidad que presentan.

\subsection{Dimensión de Hausdorff - Besicovitch}

Sea $U_{i}$ un recubrimiento (cubrimiento o cubierta) de $A$, donde el ancho de todo los intervalos $U_{i}$, satisface $w\left(U_{i}\right) \leq r$, con $w$ real. La medida $m_{r}$ está definida por la ecuación 1 de la siguiente forma:

$$
m_{r}(A)=\inf \left(\sum_{i=1}^{\infty} w\left(U_{i}\right)\right),
$$

donde el ínfimo (o el más grande de los valores mínimos) se toma sobre todos los $r$-cubrimientos de $A$.

La dimensión de Hausdorff - Besicovitch $D_{H-B}$ de $A$ se define por la ecuación 2 como:

$$
D_{H-B}=\lim _{r \rightarrow 0} m_{r}(A)
$$

\section{Dimensión Fractal por Conteo de Cajas (Box-Counting)}

Este método, conocido tambien como dimensión de capacidad, es la forma práctica más utilizada para estimar la dimensión fractal de un objeto. La mecánica de esta técnica consiste en realizar un mallado regular superpuesto a un conjunto fractal, caracterizado por el paso de malla (lado o diagonal de la celda que define la malla). Se cuenta el número de celdas que recubren a ese conjunto fractal. Variando el paso de la malla se realizan diferentes recubrimientos del conjunto fractal donde $\epsilon$ es el paso de la malla (escala) y $N(\epsilon)$ el número de celdas que recubren o interceptan en parte el conjunto fractal $F$. La ecuación 3 define la dimensión fractal por conteo de cajas $D_{B}$ como:

$$
D_{B}=\lim _{\varepsilon \rightarrow 0} \frac{\log (N(\varepsilon))}{\log (1 / \varepsilon)}
$$

La existencia de una dimensión fractal única se demuestra con una buena correlación de la recta de regresión de los puntos $\log (N(\varepsilon))$ respecto $\log (1 / \varepsilon)$. En el caso de fenómenos turbulentos, el hecho de que se desarrolle una cascada de energía entre las escalas de la producción y las escalas donde se produce la disipación, (que ocurre de forma distinta si el flujo dominante es tridimensional o bidimensional, donde se produce también una cascada inversa de energía), favorece la aparición de estructuras fractales.

\section{Regiones HII}

El espacio interestelar no se encuentra en su totalidad vacío, pues entre las estrellas existe gas neutro en diferentes estados de ionización así como granos de polvo, siendo estos últimos los principales causantes de la extinción de la radiación. Este gas está compuesto esencialmente de hidrógeno y helio y no se encuentra uniformemente distribuido, por lo 
cual en ciertas zonas de las galaxias el gas es más denso y tiene una baja temperatura, encontrándose en forma de gas neutro o molecular, mientras que en algunos otros lugares el gas se encuentra ionizado.

Esta última clase de regiones, son zonas en las que la formación estelar ha tenido lugar en los últimos millones de años, albergando estrellas masivas, de tipo espectral O y B localizadas en la secuencia principal. Estos objetos son conocidos como regiones HII, nombre propuesto por Strömgren en 193924 para designar el volumen de una nube de gas con gran cantidad de hidrógeno ionizado por una estrella caliente (o un cúmulo de estrellas calientes) con temperaturas efectivas del orden de $T_{\text {eff }}=25000-$ $50000 \mathrm{~K}$.

Dichas estrellas masivas emiten una fracción importante de su radiación como fotones lo suficientemente energéticos para provocar la fotoionización de los elementos del gas circundante. Los átomos de hidrógeno son ionizados por la radiación estelar ultravioleta $\left(U_{\mathrm{v}}\right)$ a través de fotones con energías iguales o mayores a $13.6 \mathrm{eV}$. Los electrones creados en el proceso de ionización pueden recombinarse, originando la aparición de líneas de emisión (como las series de Balmer o Paschen) que no sólo se detectan en estas regiones sino también en otros tipos de nebulosas gaseosas, como son por ejemplo las nebulosas planetarias, las cuales comparten propiedades espectrales con las regiones HII.

Uno de los inconvenientes que tiene el estudio de este tipo de regiones en galaxias externas radica en que debido a las distancias involucradas, las regiones con tamaño y brillo similares a las de la nebulosa de Orión son intrínsecamente débiles, lo cual las hace difíciles de detectar desde observatorios terrestres. Por consiguiente las regiones HII que se observan en otras galaxias son de gran tamaño, del orden de cientos de parsec y mucho más brillantes que la mayoría de las que se pueden observar en la Vía Láctea. A este tipo de objetos se les denomina regiones HII Gigantes, las cuales adicionalmente presentan una masa ionizada del orden de $10^{3}-10^{6}$ $\mathrm{M}_{\odot}$ y que a diferencia de las regiones HII normales no son ionizadas por estrellas aisladas, sino por grandes conglomerados o cúmulos de estrellas calientes, siendo su número del orden de $10^{5}$ estrellas ionizantes.

\section{Hubble V y Hubble X}

Estas dos nebulosas son objetos astronómicos que forman parte del gran complejo de regiones HII que se encuentra ubicados al norte de la barra de NGC 6822, donde adicionalmente están las regiones Hubble I y II 25, 26], así como varias asociaciones OB relativamente luminosas 27,28.

Hubble $\mathrm{V}$ y Hubble $\mathrm{X}$ tienen un tamaño en el óptico de 112 pc y 143 pc respectivamente [28, 30], siendo las dos regiones HII más luminosas de NGC 6822 y se encuentran separadas entre sí por una distancia de 320 pc. En la figura 1 se aprecia la imagen obtenida con el Telescopio Espacial Hubble de NGC 6822, en la cual se identifican las regiones HII mencionadas. Cada recuadro abarca una región HII. Hubble V se encuentra en la parte derecha de la imagen, mientras que Hubble $\mathrm{X}$ se ubica en la parte izquierda.

Se han conducido diversos estudios en el rango del óptico que describen la morfología de estas regiones. De particular interés es la fotometría de regiones HII, debido al uso potencial que tiene el emplear Regiones HII Gigantes Extra-galácticas en la determinación de distancias a escala extragaláctica. Kennicutt 31 obtuvo flujos fotoeléctricos en $\mathrm{H} \alpha$ para Hubble V y Hubble X, mientras que Hodge y colaboradores 28, 32 empleando ya tecnologías con $\mathrm{CCD}$, determinaron el flujo de $\mathrm{H} \alpha$ para 145 regiones. Los mapas de flujos de emisión en $\mathrm{H} \alpha$ de Hubble V y $\mathrm{X}$ son presentados en el trabajo de 32 como mapas

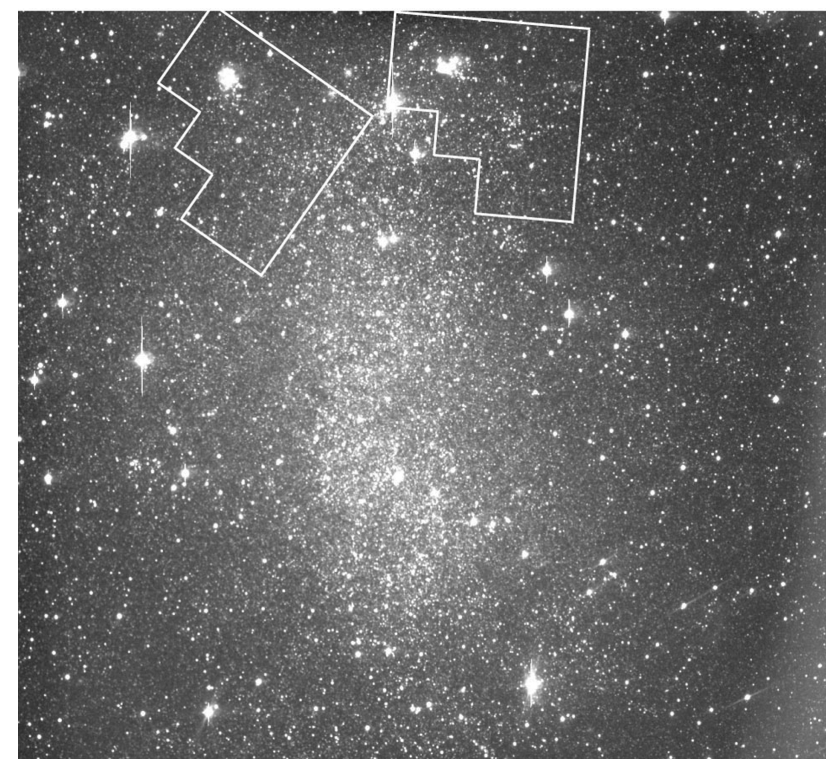

Figura 1: Imagen de 68" × 630" de NGC 6822 centrada a $19^{h} 44^{m} 54^{s}, 14^{\circ} 47^{\prime} 39^{\prime \prime}$ (E2000) obtenida con un filtro $\mathrm{V}$ [29]. La región que comprende a Hubble $\mathrm{V}$ se observa en la parte superior derecha de la imagen (oeste), mientras que Hubble $X$ se ubica en la parte superior izquierda (este). El norte está hacia arriba. 
de isocontornos del brillo de estas regiones. Recientes trabajos realizados por Odell y Bianchi [29,33] hacen uso del Telescopio Espacial Hubble (HST) y presentan una luminosidad en $\mathrm{H} \alpha$ de $4.8 \times 10^{-12}$ erg $\mathrm{cm}^{-2} \mathrm{~s}^{-1}$ para Hubble V y $3.8 \times 10^{-12} \mathrm{erg} \mathrm{cm}$ ${ }^{-2} \mathrm{~s}^{-1}$ para Hubble X. 34 presentan los primeros mapas de emisión en $H \alpha$ construidos a partir de observaciones discretas realizadas con la técnica de rendija larga. [29] concluye a través de estas observaciones que estas regiones son ópticamente gruesas y propone que el brillo de las regiones HII en $\mathrm{H} \alpha$ puede ser útil para cuantificar la radiación ultravioleta y por lo tanto la tasa de formación estelar.

Estudios sobre la cinemática de estas dos regiones se presentan en los trabajos de 27 y [35] quienes reportan velocidades radiales de ${ }^{12} \mathrm{CO}$ y 30 que presenta mapas del campo de velocidad radial de Hubble V y Hubble X en $\mathrm{H} \alpha$. La turbulencia del gas en estas nebulosas ha sido estudiada por 36,37 y [38], todo desde una perspectiva de espectros integrados y obteniendo como resultado más relevante que la velocidad de dispersión $\sigma$ presenta un comportamiento cercano al límite supersónico. 39,40 realizan estudios de la estructura interna y los gradientes de densidad de estas dos regiones HII gigantes.

\section{Análisis Fractal}

La imágenes empleadas para determinar la dimensión fractal de estas regiones HII fueron obtenidas con el Telescopio Espacial Hubble (HST) y están disponibles a través del Hubble Heritage Team. Las observaciones de Hubble $\mathrm{V}$ se realizaron el 18 de junio de 1996, con los filtros F300W (U), F487N $\left(\mathrm{H}_{\beta}\right)$, F502N ([O III]), F547M (Strömgren y), F656N $\left(\mathrm{H}_{\alpha}\right), \mathrm{F} 658 \mathrm{~N}([\mathrm{~N} \mathrm{II}])$ y el 2 de agosto de 1997 con el filtro F555W (V), con tiempo de exposición de 3.4 horas. Las observaciones de Hubble X fueron realizadas el 7 de septiembre de 1997, usando los filtros F656N $\left(\mathrm{H}_{\alpha}\right), \mathrm{F} 502 \mathrm{~N}$ ([O III] $)$ y F $487 \mathrm{~N} \mathrm{H}_{\beta}$, con tiempo de exposición de 2.6 horas. Las observaciones se obtevieron con el instrumento WFPC2 del HST.

Para determinar dimensión fractal de Hubble $\mathrm{V}$ y Hubble X, se convierten las imágenes a binario, por lo cual tan solo se aprecian dos colores : negro (valor de gris 0) y blanco (valor de gris 255). Como segunda etapa, las imágenes en binario se procesan para obtener el borde o esqueleto de cada región HII. El proceso de tranformación de las imágenes (binarización) y selección del borde o esqueleto se realizó con el software ImageJ [41.

En las figuras 2 y 3 se presentan las imágenes de Hubble X y Hubble V captadas por el Telecopio Espacial Hubble, las mismas imágenes en binario y su borde o esqueleto.

La dimensión fractal por el método de conteo de cajas se calcula con el plugin FracLac V.2.5 42] (FracLac 2015Sep090313a9330 para Hubble V y FracLac 2014Aprb1012 para Hubble X), es cual es una aplicación desarrollada en Java por Audrey Karperien 43 para ImageJ.

Las figuras 4 y 5 se presenta la variación en los tamaño de algunas de las celdas de la cuadrícula para una misma posición de la cuadrícula. Este procedimiento se realiza para 12 posiciones aleatorias de la cuadrícula. En la imagen de Hubble X, se emplearon
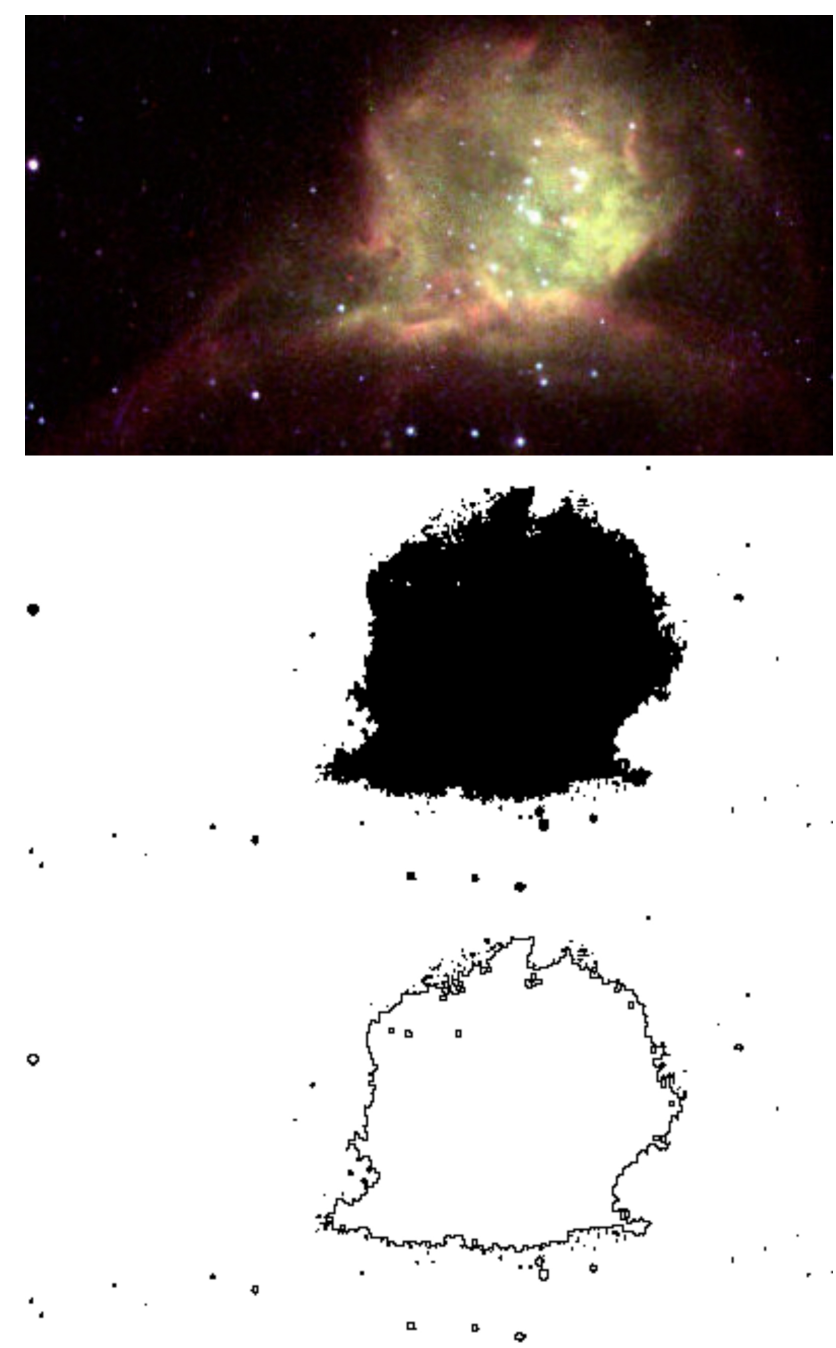

Figura 2: Imagen de Hubble $X$ captada por el telescopio espacial Hubble, en binario y su esqueleto. 

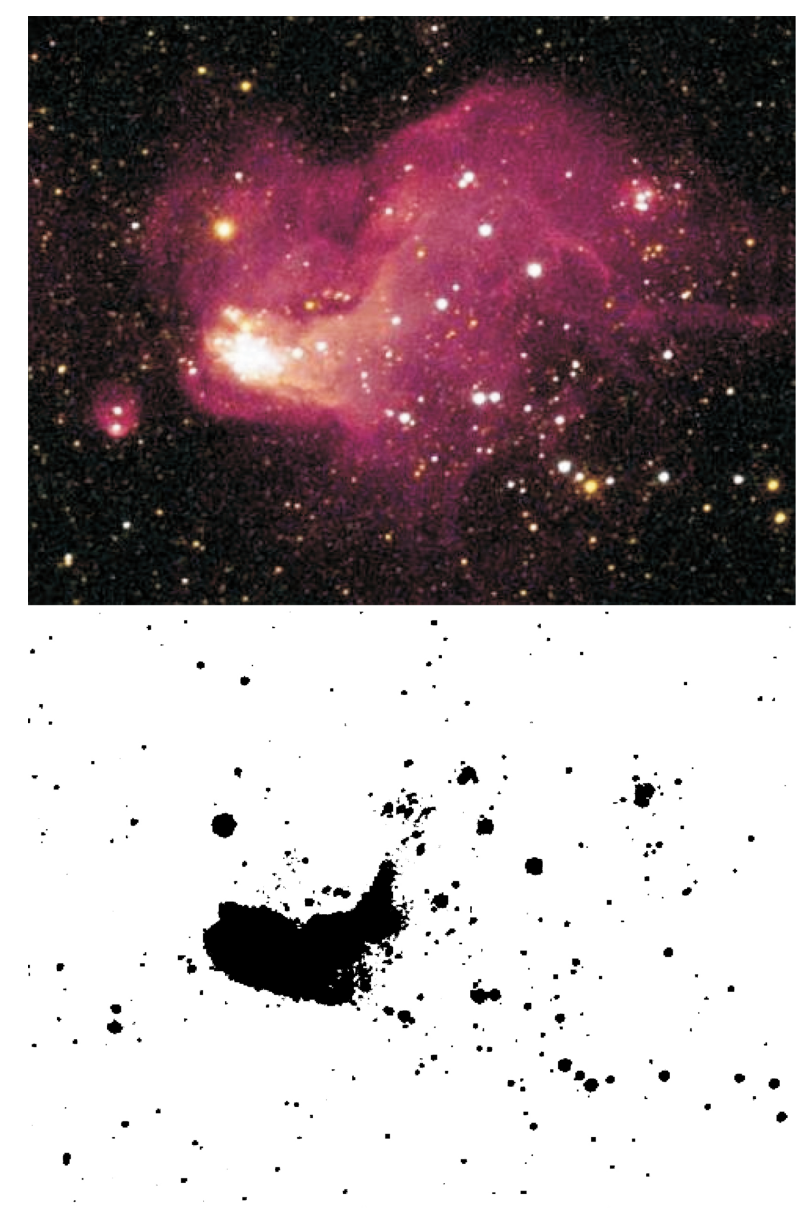

Figura 3: Imagen de Hubble V captada por el Telescopio Espacial Hubble, en binario y su esqueleto.

90 tamaños diferentes de las celdas, mientras que para la imagen de Hubble $\mathrm{V}$ fueron 100.

\section{Resultados}

En las figuras 6 y 7 se presentan los resultados obtenidos después de aplicar el método de conteo de cajas sobre la imágen de Hubble V y Hubble X res-
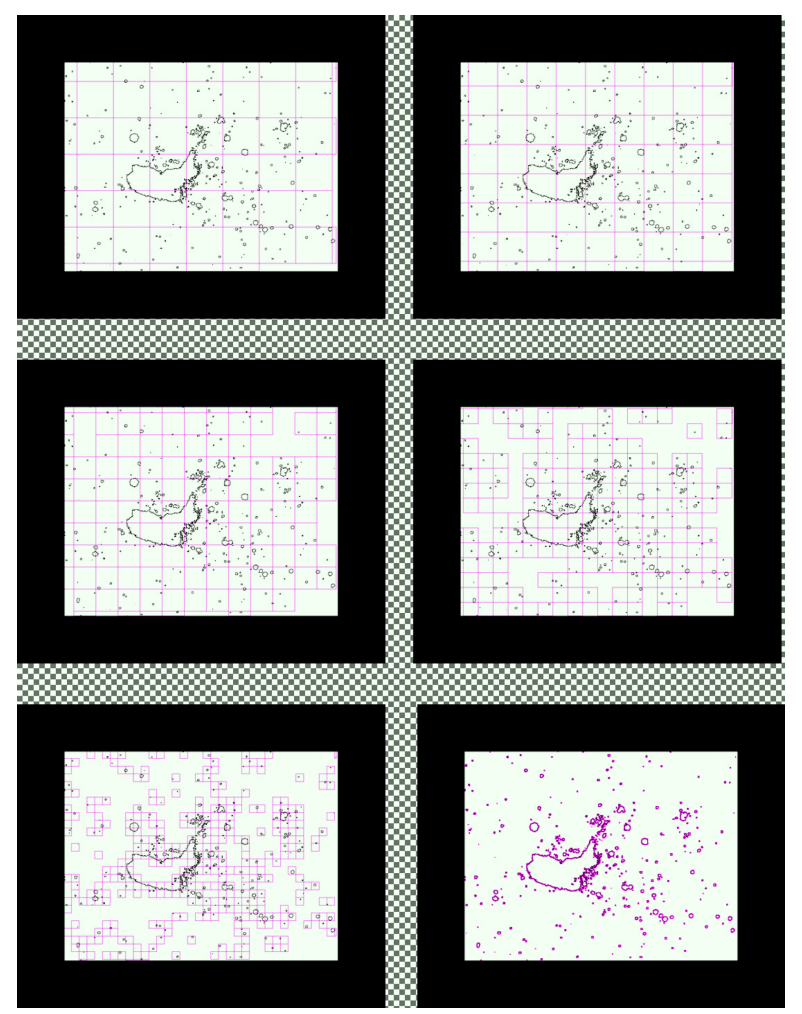

Figura 4: Algunas cuadrículas para determinar la dimensión fractal por Box Counting usando FracLac en Hubble V.
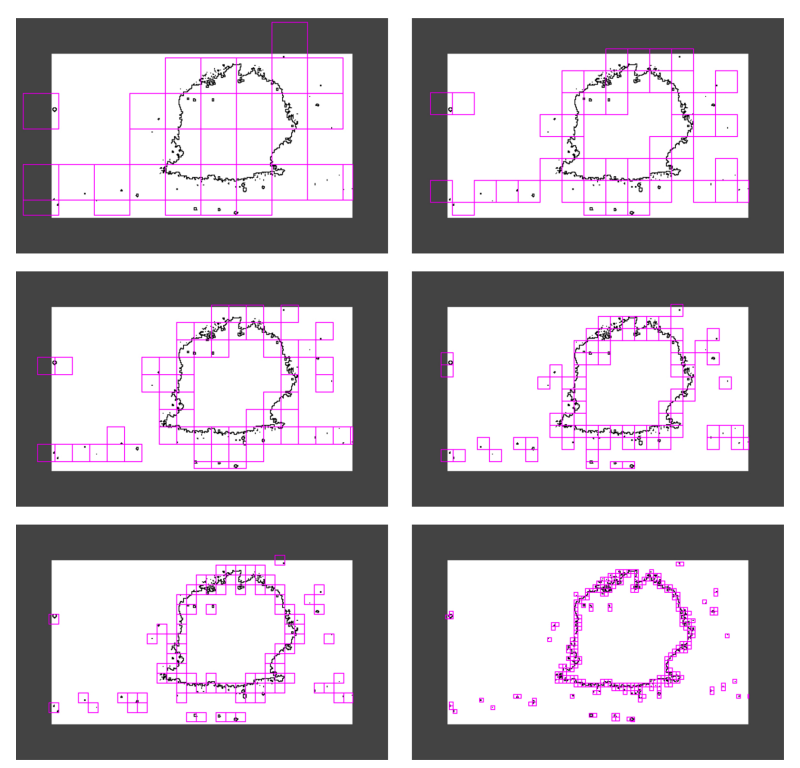

Figura 5: Algunas cuadrículas para determinar la dimensión fractal por Box Counting usando FracLac en Hubble X.

pectivamente. En la gráfica de la derecha se presenta la variación del número de cajas $N(\epsilon)$ en función del tamaño $\epsilon$ de su arista o celda. En la gráfica de la izquierda se presenta $\ln (N(\epsilon))-\ln (1 / \epsilon)$ con su respectivo ajuste lineal reaizado con el método de mínimos cuadrados. 

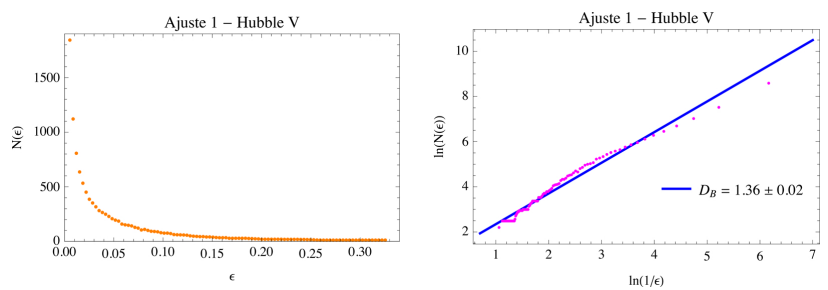

Figura 6: Graficos del Número de cajas $N(\epsilon)$ vs tamaño de caja $\epsilon$ y $\ln (N(\epsilon))-\ln (1 / \epsilon)$ para una las doce diferentes posiciones de la cuadrícula sobre la imagen de Hubble V.
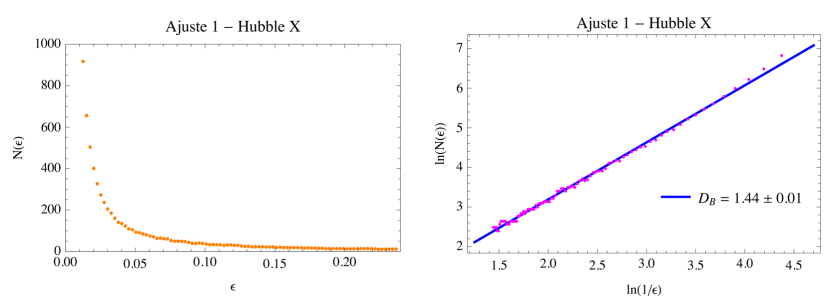

Figura 7: Graficos del Número de cajas $N(\epsilon)$ vs tamaño de caja $\epsilon$ y $\ln (N(\epsilon))-\ln (1 / \epsilon)$ para una las doce diferentes posiciones de la cuadrícula sobre la imagen de Hubble $X$.

Los valores de la pendiente de la curvas de $\ln (N(\epsilon))-$ $\ln (1 / \epsilon)$ obtenidas con el método de mínimos cuadrados corresponden a la dimensión fractal $D_{B}$ de cada una de las regiones HII. Estas se presentan en la tabla 1. acompañadas del factor de correlación $r$ y la desviación estandar $\sigma$.

La pendiente obtenida en cada curva corresponde a una medida de la dimensión fractal asociada a su respectiva region HII para una determinada posición del enmalladado sobre la imagen. Para cada imagen, se realizaron 12 mediciones de dimensión fractal, cada una con una posición diferente del enmallado.

\section{Discusión}

Desde el punto de vista geométrico, la dimensión fractal es una medida de la irregularidad o fragmentación del sistema. A medida que el valor de la dimensión fractal incrementa, el grado de irregularidad del sistema es mayor. El valor de la dimensión fractal de 1,41 $\pm 0,03$ para el Hubble X y 1,31 $\pm 0,04$ para el Hubble $\mathrm{V}$ sugieren que estas regiones HII presentan estas propiedades. Otra característica relevante e intrinseca en las regiones HII es la turbulencia, la cual tiene asociada una geometría que se manifiesta precisamente en la forma de la frontera y del espacio en la que se produce disipación de energía. Zhao Yuxin et al [44], sugiere que en la transición de un flujo laminar a uno turbulento la dinámica y el contorno se rompen por completo, presentandose irregularidades en el borde del sistema, la cual puede ser evaluada por la dimensión fractal [44. Por lo tanto, los valores de dimensión fractal obtenidos en este trabajo indican que los procesos de turbulencia al interior de estas dos regiones no son grandes. Esto coincide con lo reportado por Caicedo y colaboradores [45], donde se obtiene que la velocidad de dispersión ( que es la cantidad que cuantifica la turbulencia ) para Hubble V y Hubble $\mathrm{X}$ es de $10 \pm 2 \mathrm{~km} / \mathrm{h}$ en la linea de emisión de $\mathrm{H} \alpha$, siendo un valor límite entre un comportamiento sonico-subsonico.

La tabla 2 presenta la dimensión fractal de Hubble $\mathrm{V}$ y Hubble $\mathrm{X}$ encontrada en este trabajo, así como de otras nebulosas reportadas en la literatura. Los pocos estudios existentes sobre dimensión fractal en regiones HII [46-49] únicamente calculan esta cantidad sin especificar la metodología ni

Tabla 1: Dimensión fractal de Hubble $V$ y Hubble $X$.

\begin{tabular}{|c|c|c|c|c|c|c|}
\hline \multirow{2}{*}{ Ajuste } & \multicolumn{3}{|c|}{ Hubble V } & \multicolumn{3}{c|}{ Hubble X } \\
\cline { 2 - 7 } & $D_{B}$ & $r^{2}$ & $\sigma_{\text {est }}$ & $D_{B}$ & $r^{2}$ & $\sigma_{\text {est }}$ \\
\hline \hline 1 & $1,36 \pm 0,02$ & 0,9758 & 0,2078 & $1,44 \pm 0,01$ & 0,9967 & 0,0597 \\
\hline 2 & $1,35 \pm 0,02$ & 0,9741 & 0,2144 & $1,45 \pm 0,01$ & 0,9971 & 0,0569 \\
\hline 3 & $1,35 \pm 0,02$ & 0,9756 & 0,2074 & $1,46 \pm 0,01$ & 0,9978 & 0,0496 \\
\hline 4 & $1,35 \pm 0,02$ & 0,9744 & 0,2135 & $1,43 \pm 0,01$ & 0,9968 & 0,0586 \\
\hline 5 & $1,30 \pm 0,02$ & 0,9796 & 0,1820 & $1,39 \pm 0,01$ & 0,9954 & 0,0690 \\
\hline 6 & $1,29 \pm 0,02$ & 0,9780 & 0,1889 & $1,39 \pm 0,01$ & 0,9950 & 0,0717 \\
\hline 7 & $1,29 \pm 0,02$ & 0,9771 & 0,1916 & $1,39 \pm 0,01$ & 0,9950 & 0,0713 \\
\hline 8 & $1,29 \pm 0,02$ & 0,9795 & 0,1809 & $1,39 \pm 0,01$ & 0,9949 & 0,0715 \\
\hline 9 & $1,29 \pm 0,02$ & 0,9757 & 0,1984 & $1,39 \pm 0,01$ & 0,9947 & 0,0734 \\
\hline 10 & $1,30 \pm 0,02$ & 0,9778 & 0,1902 & $1,39 \pm 0,01$ & 0,9951 & 0,0714 \\
\hline 11 & $1,29 \pm 0,02$ & 0,9794 & 0,1817 & $1,40 \pm 0,01$ & 0,9952 & 0,0703 \\
\hline 12 & $1,28 \pm 0,02$ & 0,9794 & 0,1807 & $1,39 \pm 0,01$ & 0,9947 & 0,0737 \\
\hline$D_{\text {Brom }}$ & \multicolumn{3}{|c|}{$1,31 \pm 0,04$} & \multicolumn{5}{c|}{$1,41 \pm 0,03$} \\
\hline
\end{tabular}


técnicas computacionales empleadas y solo asocian este comportamiento de las nebulosas al observado y cuantificado en otras estructuras fractales.

Si se considera que la dimensión fractal es un indicador indirecto del grado de turbulencia en las regiones HII, una clasificación basada esta propiedad ofrece como ventaja el poder estudiar distintas nebulosas de este tipo a partir unicamente de las imágenes en el visible obtenidas por un telescopio óptico, como por ejemplo el Telescopio Espacial Hubble. En el campo educativo y pedagógico, esta metodología puede ser extendida y aplicada a otros objetos astronómicos como nebulosas planetarias, galaxias. Los estudiantes en asignaturas de física y matemáticas a nivel de licenciatura pueden construir pequeños catálogos de objetos astronómicos basado en la dimensión fractal y correlacionarlos con otros catálogos que hacen referencia a otras características de los mismos objetos y buscar una correlacion entre ellos.

\section{Conclusiones}

La introducción de la geometría fractal en el campo de la astrofísica, amplia el panorama de las investigaciones teóricas-experimentales en este campo. Empleando el método de conteo de cajas, es posible cuantificar la dimensión fractal de complejas estructuras espaciales, como las regiones HII aquí estudiadas. Esta metodología no es exclusiva de nebulosas gaseosas irregulares y puede ser aplicada como herramienta para la clasificación de las galaxias irregulares, manchas solares, cráteres de impactos, etc.

La dimensión fractal de Hubble $\mathrm{V}$ y Hubble $\mathrm{X}$ es $1,31 \pm 0,04$ y $1,41 \pm 0,03$ respectivamente, que nos indica un bajo comportamiento en la estructura irregular de estas regiones HII. Al emplear los datos reportados en la literatura y proponer una clasifi- cación basada en esta propiedad, se evidencia este comportamiento.

El análisis por dimensión fractal es particularmente apropiado para estudiar sistemas jerarquicos autosimilares. Esta dimensión es un parámetro estructural distintivo. Debido a que la dimensión fractal está definida en términos de un logaritmo de escala, este sería único para objetos astronómicos observados en un cierto rango de longitudes de onda. Para generar un ligero cambio en su dimensión fractal, un objeto astronómico debe cambiar sus dimensiones en enormes proporciones, lo cual, para las regiones HII aquí estudiadas ocurre a grandes escalas de tiempo.

Aunque este es un primer paso hacia el análisis de estructuras astronómicas como las regiones HII empleando técnicas de los sistemas complejos, no se puede considerar como el marco "final" para describir los patrones y propiedades de objetos astronómicos, pero si como una nueva herramienta para clasificar estos objetos. El hecho de que uno de estos objetos sea un fractal, es porque es observado a través de una pequeña gama de longitudes de onda. Una completa descripción bajo el formalismo de la geometría fractal requerirá observaciones en todo los rangos de longitudes de onda posible.

Desde el punto de vista pedagógico, el uso de la dimensión fractal como un nuevo parámetro de clasificación ha sido poco explotado. La metodología que presentamos en este trabajo permite mostrar al estudiante contenidos que no se encuentran en los planes de estudio convencionales de física, con casos concretos o inusualmente tratados. Es posible replicar este estudio en asignaturas avanzadas de física y matemáticas como nuevas propuestas de pequeños proyectos de investigación y/o profundización con otros objetos astronómicos de gran interés como galaxias, cráteres en la luna, manchas solares, nebulosas planetarias y exoplanetas (para las cuales

Tabla 2: Dimensión fractal de diferentes objetos astronómicos. Valores obtenidos en este trabajo [e].

\begin{tabular}{|c|c|c|}
\hline Objeto Astronómico & $D_{B}$ & Referencia \\
\hline Remanente de la Supernova de Vela & $1,2 \pm 0,05$ & {$[\overline{46}$} \\
\hline Nebulosa de Eta Carinae & $1,37 \pm 0,02$ & $\overline{46}$ \\
\hline Región HII Gigante Hubble V & $1,31 \pm 0,04$ & {$[\mathrm{e}]$} \\
\hline Región HII GiganteHubble X & $1,41 \pm 0,03$ & [e] \\
\hline Nebulosa Cabeza de Caballo & 1,6965725 & 47 \\
\hline Paley I & 1,677181 & $\overline{47}$ \\
\hline Nebulosa Rosette & 1,78261 & 47 \\
\hline Nebulosa del Aguila & 1,669622 & $\overline{47}$ \\
\hline IC434 (col I) & 1,811718 & 48 \\
\hline Nebulosa de la Hélice (NGC 7293) & 1,841059 & $\overline{50}$ \\
\hline
\end{tabular}


existe gran cantidad de datos científicos de libre acceso disponible en la red).

\section{Referencias}

[1] R.A. Meyers, Encyclopedia of complexity and systems science (Springer, 2009).

[2] B. Mandelbrot, Les Objets Fractals (The Fractal Objects) (Flammarion, Paris, France, 1975), p. 246.

[3] A. Heck, J.M. Perdang, Applying fractals in astronomy (Springer-Verlag, Berlin Heidelberg, 1991).

[4] G.De Vaucouleurs, Science 167, 1203 (1970).

[5] G. De Vaucouleurs, Publications of the Astronomical Society of the Pacific 83, 113 (1971).

[6] G. De Vaucouleurs, in The Formation and Dynamics of Galaxies, edited by J.R. Shakeshaft (Springer, Netherlands, 1974).

[7] M. Davis, P. Peebles, ApJ 267, 465 (1983).

[8] B.J. Jones, V.J. Martinez, E. Saar, J. Einasto, ApJ 332, L1 (1988).

[9] N.A. Bahcall, R.M. Soneira, ApJ 270, 20 (1983).

[10] N.A .Bahcall, ARA\&A 26, 631, (1988).

[11] H. Rood, ARA\&A 26, 245, (1988).

[12] P.J.E. Peebles, The large-scale structure of the universe. (Princeton University Press, New Jersey, 1980).

[13] P. Peebles, Physica D: Nonlinear Phenomena 38, 273, (1989).

[14] P.H. Coleman, L. Pietronero, Physics Reports 213, 311, (1992).

[15] P. Coleman, L. Pietronero, R. Sanders, A\&A 200, L32, (1988).

[16] S. Yoshioka, S. Ikeuchi, ApJ 341, 16, (1989).

[17] V. Martínez, in Applying fractals in astronomy, edited by A. Heck, J.M. Perdang, (Springer-Verlag, Berlin Heidelberg, 1991).

[18] J. Serra, Image analysis and mathematical morphology, (Academic Press, Inc. Orlando, 1983), v. 1.

[19] J. Perdang, Vistas in Astronomy 33, 249 (1990).

[20] J. Dubois, La dynamique non linéaire en physique du globe ( Elsevier / Masson, Paris, 1995), p.265.

[21] F. Hausdorff, Mathematische Zeitschrift 3, 314 (1919).

[22] A. Besicovitch, Mathematische Annalen 101, 161 (1929).

[23] H. Hurst, Nature 180, 494 (1957).

[24] B. Strömgren, ApJ 89, 526 (1939).

[25] E.P. Hubble ApJ 62, 409 (1925).

[26] P. Hodge, M.G. Lee, R.C Jr. Kennicutt, PASP 100, 917 (1988).

[27] C.D. Wilson, AJ 104, 1374 (1992).

[28] P. Hodge, M.G. Lee, R.C. Jr. Kennicutt, PASP 101, 32 (1989).

[29] C.R. O’Dell, P.W. Hodge, R.C. Jr. Kennicutt, PASP 111, 1382 (1999).

[30] A. Tomita, K. Ohta, M. Saito, PASJ 45, 693 (1993).
[31] R.C. Jr. Kennicutt, ApJ 228, 704 (1979).

[32] J. Collier, P. Hodge, ApJS 92, 119 (1994).

[33] L. Bianchi, S. Scuderi, P. Massey, M. Romaniello, AJ 121, 2020 (2001).

[34] H.E. Caicedo-Ortiz, H. Castañeda, RevMexAA (Conference Series) 40, 187 (2011).

[35] F.P. Israel, F. Baas, R.J. Rudy, E.D. Skillman, C.E. Woodward, A\&A 397, 87 (2003).

[36] H.H. Hippelein, A\&A 160, 374 (1986).

[37] J. Melnick, M. Moles, R. Terlevich, J.M. GarciaPelayo, MNRAS 226, 849 (1987).

[38] J.R. Roy, R. Arsenault, ApJ 302, 579 (1986).

[39] J. Pérez Oregón, H.E. Caicedo-Ortiz,H. Castaneda, A. Hidalgo-Gámez, Jou.Cie.Ing. 6, 16 (2014).

[40] H.E. Caicedo Ortiz, H. Castañeda, J. Pérez Oregón, RevMexAA (Conference Series) 44, 16 (2014).

[41] ImageJ, Available from: https://imagej .nih.gov/ ij/download.html

[42] A. Karperien, Fraclac, Available from: http://rsb.info.nih.gov/ij/plugins/ fraclac/Frac_Lac.jar.

[43] A. Karperien, FracLac for ImageJ. US National Institutes of Health [online] (2013).

[44] Y. Zhao, S. Yi, L. Tian, L. He, Z Cheng, Science in China Series G: Physics, Mechanics and Astronomy 51, 1134 (2008).

[45] H.E. Caicedo-Ortiz, E. Santiago-Cortés, J. LópezBonilla, H. Castañeda, Journal of Physics: Conference Series 582, 012049 (2015).

[46] S. Blacher, J. Perdang, Vistas in Astronomy 33, 393 (1990).

[47] S. Datta, arXiv:astro-ph/0105036 (2001).

[48] S. Datta, A\&A 401, 193 (2003).

[49] B.G. Elmegreen, S. Kim, L. Staveley-Smith, ApJ 548, 749 (2001).

[50] S. Datta, in Planetary Nebulae: Their Evolution and Role in the Universe, edited by S. Kwok, M. Dopita, R. Sutherland (Astronomical Society of the Pacific, 2003), v. 209, p. 523. 